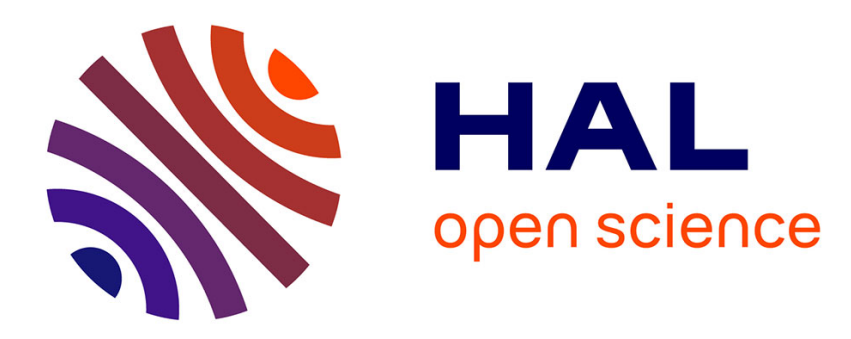

\title{
Propagation et génération des streamers dans les diélectriques liquides
}

\author{
A. Beroual, R. Tobazeon
}

\section{To cite this version:}

A. Beroual, R. Tobazeon. Propagation et génération des streamers dans les diélectriques liquides. Revue de Physique Appliquée, 1987, 22 (9), pp.1117-1123. 10.1051/rphysap:019870022090111700 . jpa-00245653

\section{HAL Id: jpa-00245653 https://hal.science/jpa-00245653}

Submitted on 1 Jan 1987

HAL is a multi-disciplinary open access archive for the deposit and dissemination of scientific research documents, whether they are published or not. The documents may come from teaching and research institutions in France or abroad, or from public or private research centers.
L'archive ouverte pluridisciplinaire HAL, est destinée au dépôt et à la diffusion de documents scientifiques de niveau recherche, publiés ou non, émanant des établissements d'enseignement et de recherche français ou étrangers, des laboratoires publics ou privés. 


\title{
Propagation et génération des streamers dans les diélectriques liquides
}

\author{
A. Beroual et R. Tobazeon \\ C.N.R.S., Laboratoire d'Electrostatique et de Matériaux Diélectriques (*), 166 X, 38042 Grenoble Cedex, \\ France
}

(Reçu le 24 novembre 1986, révisé le 14 avril 1987, accepté le 18 mai 1987)

\begin{abstract}
Résumé. - Cette étude concerne la génération et la propagation des streamers en géométrie pointe-plan sous créneau de tension. Un nouveau dispositif expérimental a permis de montrer qu'il existe une relation étroite entre la forme du streamer, sa vitesse de propagation, la forme du courant et la lumière qu'il émet qu'elles que soient la polarité de la pointe et la nature du liquide. Plus le streamer est filamentaire, plus il est rapide et réciproquement. La distribution de vitesse admet un minimum pour un très grand nombre de liquides isolants pur ou renfermant divers additifs. L'amplitude des vitesses dépend d'une manière importante des propriétés électroniques du liquide et des additifs. L'effet de la pression hydrostatique sur l'apparition des streamers dans les deux polarités, indique l'existence d'une phase gazeuse. Il semble que le mécanisme de cavitation induite par des mouvements électrohydrodynamiques résultant d'une injection unipolaire de charges ne soit pas à l'origine de cette phase gazeuse.
\end{abstract}

\begin{abstract}
This study concerns the initiation and propagation of streamers in point-plane geometry. Our experimental set-up has permitted us to show the existence of a close correlation between the shape of the streamer, its propagation velocity, the current shape and the light it emits, whatever are the polarity of the point and the liquid nature. The more filamentary the streamer, the higher its velocity. The propagation velocity has a minimum for a large variety of insulating liquids wheter pure or containing different additives. The amplitude of the velocity depends strongly on the electronic properties of the liquid and additives. The effects of hydrostatic pressure on the streamer appearance for both polarities suggest the existence of a gas phase. It appears that electrohydrodynamically induced cavitation phenomena, resulting from unipolar charge injection, are not responsible for the gas phase.
\end{abstract}

\section{Introduction.}

L'utilisation de nouvelles techniques optiques a permis de réaliser d'importants progrès dans la compréhension des processus fondamentaux conduisant au claquage des diélectriques liquides. Ainsi, il est devenu possible de photographier et de suivre l'évolution des différents événements précédant le claquage. Durant cette phase de préclaquage, une perturbation appelée streamer prend naissance dans une région de champ élevé (phase de génération) et se développe vers l'électrode opposée (phase de propagation). La plupart des travaux antérieurs ont été consacrés à la phase de propagation dans un système d'électrodes pointe-plan lorsque la pointe est anode. Cependant, plusieurs points n'ont pas

(*) Laboratoire Associé à l'Univ. Scient. Techn. et Méd. de Grenoble. encore été clarifiés et certains résultats restent contradictoires.

L'objet de ce travail est d'essayer de dégager à partir d'une étude systématique sous créneau de tension (durée $\leq 55 \mu \mathrm{s}$ ) en géométrie pointe-plan dans les deux polarités, effectuée grâce à un nouveau dispositif expérimental, les résultats les plus marquants relatifs à la génération et à la propagation des streamers dans différents liquides (purs ou contenant des additifs). L'influence de la pression hydrostatique sur ces phénomènes a été également considérée.

\section{Dispositif expérimental.}

Le circuit d'essai est constitué d'un générateur de créneau de tension, d'une cellule de mesure et d'un dispositif de visualisation (Fig. 1).

2.1 Cellule de mesure. - Elle est constituée d'un corps en téflon et comporte deux hublots en 


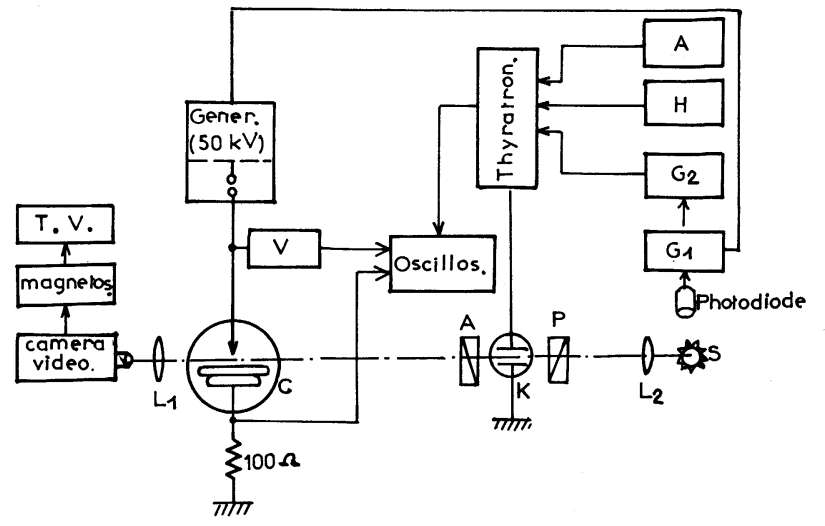

Fig. 1. - Schéma général de l'appareillage expérimental. $\mathrm{K}$ : cellule de Kerr ; $\mathbf{A}$ et $\mathbf{P}$ : analyseur et polariseur de lumière ; $\mathrm{L}_{1}$ et $\mathrm{L}_{2}$ : lentilles ; $\mathrm{C}$ : cellule d'essais ; $\mathrm{A}$ : alimentation du thyratron; $\mathbf{H}$ : chauffage du thyratron; $\mathrm{G}_{1}$ : générateur d'impulsion permettant la synchronisation de la tension et de la source de lumière $S ; G_{2}$ : générateur d'impulsion permettant d'ajuster le temps de retard à l'exposition; V : sonde haute tension.

[Schematic diagram of the experimental apparatus. $\mathbf{K}$ : KERR cell ; $A$ and $P$ : light analyser and polarizer ; $L_{1}$ and $L_{2}$ : lenses ; $C$ : test cell ; $A$ : thyratron power supply ; $\mathrm{H}$ : thyratron heating; $\mathrm{G}_{1}$ : pulse generator for the synchronization of the applied voltage and the spark light $S ; \mathrm{G}_{2}$ : pulse generator to adjust the exposure time delay ; V : high voltage probe.]

pyrex de $10 \mathrm{~mm}$ de diamètre et un système d'électrodes pointe-plan. L'électrode plane en laiton de $10 \mathrm{~mm}$ de diamètre peut être recouverte ou non d'une feuille d'isolant solide (polypropylène) de $100 \mu \mathrm{m}$ d'épaisseur. L'intérêt d'employer cet isolant (dans la mesure où il ne claque pas) est qu'il empêche l'apparition d'un arc entre les électrodes. La plupart des résultats rapportés dans ce travail ont été obtenus avec l'électrode plane recouverte d'isolant. Nous avons vérifié que ni la vitesse, ni les courants ne sont modifiés par la présence de cette feuille d'isolant.

L'électrode pointe est une aiguille en acier dur (Ogura Jewel Industry Company Limited), de rayon de courbure variable $(3$ à $100 \mu \mathrm{m})$ et de conicité $30^{\circ}$.

Les distances interélectrodes $(L)$ étudiées peuvent aller de 0,5 à $6 \mathrm{~mm}$.

Un piston (en contact avec la surface du liquide) actionné par de l'azote comprimé, permet d'obtenir des surpressions variables de 0 à 40 bars. Une pompe à vide et un système de valves sont utilisés pour travailler en pressions subatmosphériques; le minimum possible est $5 \times 10^{-2}$ bars.

L'échauffement et le refroidissement du liquide sont obtenus grâce à la circulation de glycol dans un serpentin entourant la cellule. La gamme de température est de -30 à $+70^{\circ} \mathrm{C}$.
2.2 GÉnÉRATEuR DE TENSION. - Le générateur d'échelon de tension à tube de $50 \mathrm{kV}$ (EIMAX 4 PR $250 \mathrm{C})$ maximum, construit au Laboratoire, produit une onde (durée $\leq 55 \mu$ s) qui croît linéairement de 0 à la valeur choisie. Le temps de montée de la tension $t_{\mathrm{m}}$ peut être ajusté de 0,4 à $25 \mu \mathrm{s}$.

2.3 VisuAlisation DES STREAMERS. - La visualisation est réalisée par la méthode d'ombroscopie.

Pour visualiser les streamers et mesurer leur vitesse de propagation, on illumine l'espace interélectrodes par un éclair de durée très brève et on photographie les événements qui s'y produisent. Comme nous ne disposons pas de caméra ultrarapide, nous avons développé une méthode utilisant une caméra vidéo (Philips type LDH. 0400/01) dont le tube a une rémanence suffisante pour permettre l'enregistrement sur un magnétoscope de l'allure du streamer à l'instant choisi.

L'image de l'intervalle entre électrodes est produite sur le tube de la caméra vidéo par deux lentilles $L_{1}$ et $L_{2}$. Une source de lumière à xénon produit un éclair dont la durée dépend des lampes utilisées (30 à $80 \mu \mathrm{s})$. Une cellule de Kerr alimentée par un thyratron et jouant le rôle d'obturateur de lumière, permet d'obtenir un temps d'exposition de 100 ns. Deux générateurs d'impulsions (HewlettPackard model 214 A) permettent d'ajuster le temps de retard à l'exposition $(\tau)$ par rapport au zéro de la tension.

2.4 DÉTECTION DE LA LUMIÈRE ÉMISE PAR LE STREAMER. - Elle est détectée par un photomultiplicateur (RCA type 7265) placé devant l'une des fenêtres de la cellule d'essai et dont la sortie est connectée à l'oscilloscope à travers une résistance de $100 \Omega$.

2.5 ENREGISTREMENT DES DIFFÉRENTS SIGNAUX. - La forme de la tension appliquée, le temps de retard $\tau$, le courant transitoire et la lumière émise par le streamer, sont enregistrés sur des oscilloscopes à mémoire (Tektronix 7623). La tension est mesurée par l'intermédiaire d'une sonde Tektronix PC 015.

Les courants transitoires sont déduits de la tension aux bornes d'une résistance de $100 \Omega$. Deux diodes rapides montées tête-bêche, protègent les tiroirs de l'oscilloscope contre d'éventuelles surtensions.

2.6 MÉThOde DE MESURE DE LA VITESSE DU STREAMER. - Pour un intervalle entre électrodes et une tension donnés, on mesure la longueur du streamer $l_{\mathrm{s}}$ (longueur de la branche la plus proche de l'électrode opposée) pour différentes valeurs de $\tau$ fixées au préalable. Chaque valeur est une moyenne sur vingt essais. La vitesse moyenne est déduite de la différence dans les longueurs de streamers $(v=$ $\left(l_{\mathrm{s} 2}-l_{\mathrm{s} 1}\right) /\left(\tau_{2}-\tau_{1}\right)$, où $l_{\mathrm{s}}=l_{\mathrm{s} 1}$ à $\left.\tau=\tau_{1}\right)$. L'intérêt 
de cette méthode est qu'elle ne dépend pas du temps de retard à l'apparition du streamer.

Les vitesses mesurées par notre système sont au plus égales à $10 \mathrm{~km} / \mathrm{s}$.

\section{Propagation des streamers.}

L'idée communément admise est que seuls les streamers positifs ont une forme filamentaire associée à une vitesse élevée; les streamers négatifs étant plutôt en forme de buisson. En ajoutant de faibles concentrations de composés capteurs d'électrons (chlorure d'éthylène) Devins et al. [1], ont observé que les streamers négatifs (pointe négative) devenaient filamentaires et que leurs vitesses augmentaient. La présence d'un additif à bas potentiel d'ionisation ( $\mathrm{N}-\mathrm{N}^{\prime}$ diméthylaniline : DMA) n'altère pas la vitesse des streamers négatifs mais accrôit celles des positifs. D'autre part, dans les liquides renfermant des halogènes tels le tétrachlorure de carbone $\left(\mathrm{CCl}_{4}\right)$, le chlorobenzène ou l'iodobenzène, les streamers négatifs et positifs se propagent très rapidement [2]. En effectuant une étude systématique sur le cyclohexane - liquide dans lequel les streamers négatifs sont parmi les plus lents -, nous avons pu vérifier ces résultats et élargir notre étude à d'autres liquides (alcanes, cyclohexane, hydrocarbures aromatiques, phénylxylyléthane - P.X.E. mélange de mono et dibenzyltoluène - MDBT huile de transformateur).

Généralement, nous observons une corrélation très étroite entre la forme du streamer et sa vitesse de propagation indépendamment du liquide (pur ou contenant des additifs) et de la polarité de la pointe. Plus le streamer est rapide, plus il est filamentaire et réciproquement. Quant à l'amplitude des vitesses, elle est intimement liée à la structure du liquide et en particulier aux affinités électroniques de la molécule (facilité d'attachement et de détachement d'électrons). En effet, nous avons mis en évidence que la seule présence d'un atome de chlore dans la molécule du chlorocyclohexane fait que la vitesse en polarité négative est multipliée par un facteur 8 par rapport à celle du cyclohexane [3].

L'action d'additifs capteurs d'électrons ou de composés à bas potentiel d'ionisation sur la vitesse des streamers est également remarquable. Cependant, l'effet des capteurs d'électrons sur les streamers négatifs est beaucoup plus important que celui des composés à bas potentiel d'ionisation sur les streamers positifs. En effet, l'addition de $0,04 \mathrm{M} / 1 \mathrm{de}$ $\mathrm{CCl}_{4}$ - capteur d'électrons - au cyclohexane entraîne une augmentation de la vitesse des streamers négatifs d'un facteur 10 (Fig. 2) ; les streamers deviennent filamentaires.

L'addition de $0,05 \mathrm{M} / 1$ de tétraméthylène paradiphénylamine (TMPD) - composé à bas potentiel d'ionisation - au cyclohexane provoque une

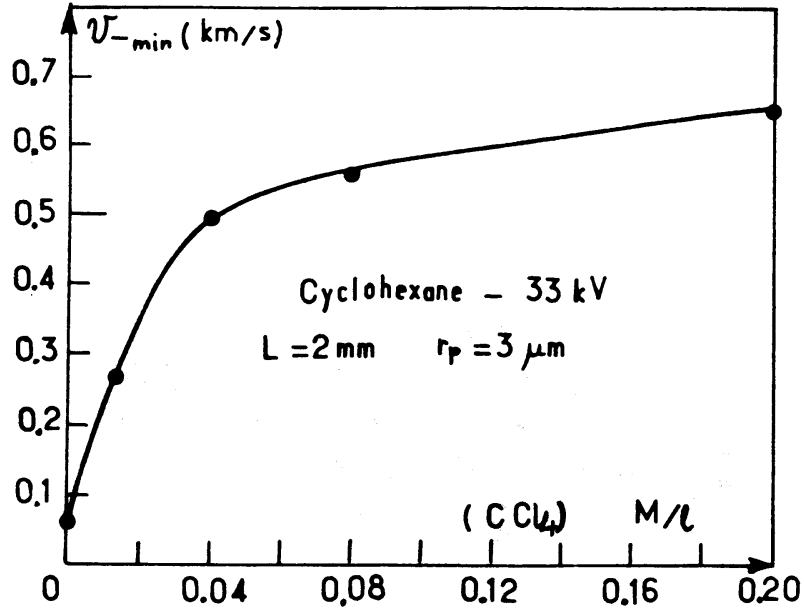

Fig. 2. - Effet d'un additif capteur d'électrons $\left(\mathrm{CCl}_{4}\right)$ sur la vitesse des streamers négatifs dans le cyclohexane.

[Effect of an electron scavenger additive $\left(\mathrm{CCl}_{4}\right)$ on the negative streamer velocity in cyclohexane.]

augmentation modérée de la vitesse des streamers négatifs (moins d'un facteur 2) dont l'allure est peu modifiée; dans les mêmes conditions celle des streamers positifs est multipliée par un facteur de l'ordre de 3 et ils deviennent plus filamentaires encore ; en présence de DMA, ce facteur ne dépasse pas 2 (Fig. 3).

D'autre part, les streamers négatifs lents sont caractérisés par des courants formés de plusieurs pics discrets (Fig. 4a) alors que les streamers positifs rapides donnent lieu à des courants dont la variation

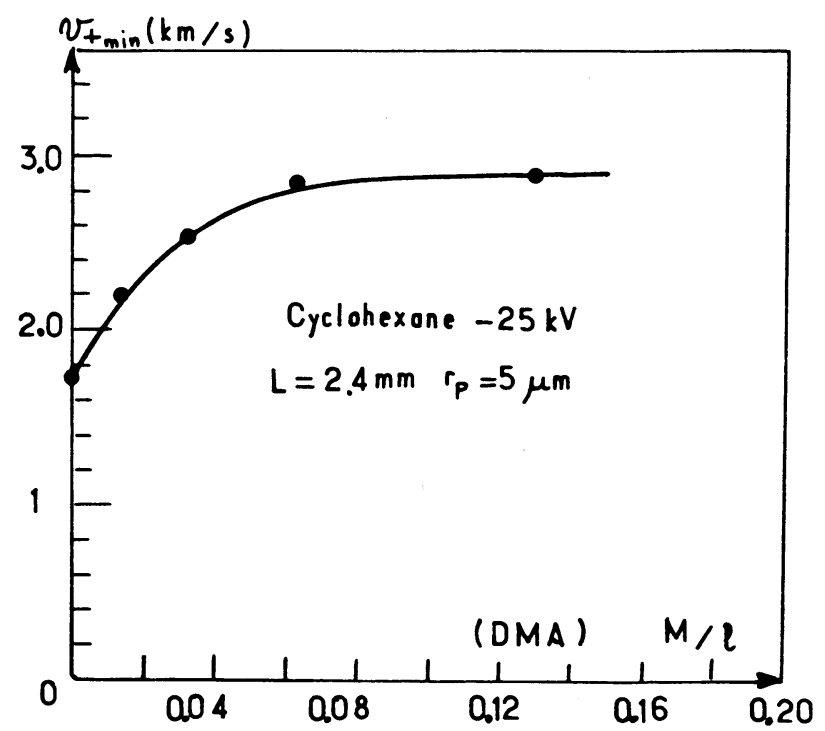

Fig. 3. - Effet d'un composé à bas potentiel d'ionisation (DMA) sur la vitesse des streamers positifs dans le cyclohexane.

[Effect of a low ionisation potentiel compound (DMA) on the positive streamer velocity in cyclohexane.] 
est assez régulière (Fig. 4b) jusqu'à un maximum correspond à l'arrivée du streamer sur l'électrode opposée ; le temps correspondant à ce maximum permet d'obtenir la vitesse moyenne du streamer [3]. De plus, les courants sont parfaitement corrélés avec
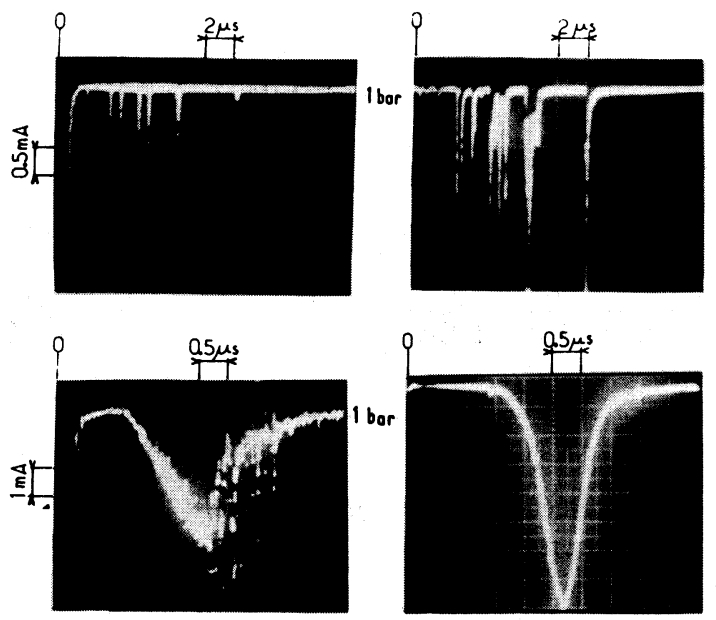

Fig. 4. - Courant et lumière émise par le streamer dans le MDBT. $L=1,5 \mathrm{~mm} ; \quad r_{\mathrm{p}}=3 \mu \mathrm{m} ; \quad U=32 \mathrm{kV}$. (a) pointe négative ; (b) pointe positive.

[Current and light emitted by the streamer in MDBT. $L=1.5 \mathrm{~mm} ; r_{\mathrm{p}}=3 \mu \mathrm{m} ; U=32 \mathrm{kV}$. (a) negative point ; (b) positive point.]

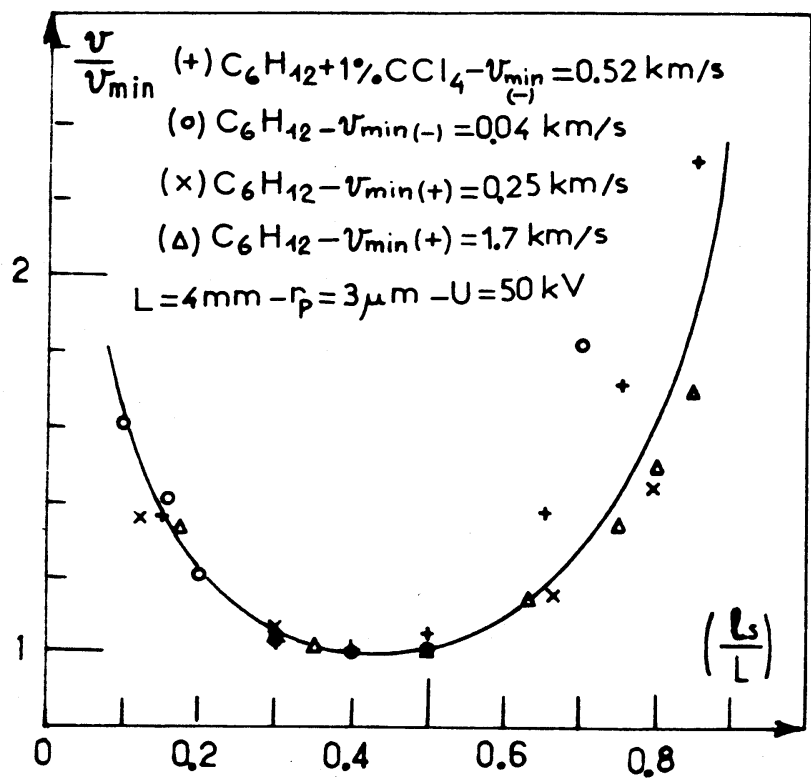

Fig. 5. - Distribution de la vitesse réduite en fonction de la longueur du streamer réduite dans le cyclohexane. La courbe pleine représente $E / E_{\min }$ (théorique) pour une sphère conductrice concentrique à la pointe pour $r_{\mathrm{p}} / L=0,1$.

[Reduced streamer velocity against the reduced streamer growth in cyclohexane. The full curve represents $E / E_{\text {min }}$ (theoretical value) for a conducting sphere concentric to the point for $r_{\mathrm{p}} / L=0.1$.] la lumière émise qui accompagne le streamer comme celà a été rapporté par d'autres chercheurs $[4,5]$.

Il existe deux modes de propagation : subsonique et supersonique pour une polarité donnée (Fig. 5). Le mode supersonique apparaissant plus fréquemment quand on augmente la tension ou/et la pression hydrostatique du liquide; lorsqu'on augmente la température on favorise l'apparition des streamers subsoniques (Fig. 6). Ce phénomène paraît général, alors qu'il n'a été observé que dans le $n$-hexane en pointe positive [5] et dans l'huile de transformateur en pointe négative [4].

La distribution des vitesses admet toujours un minimum plus ou moins marqué situé entre le milieu et les $2 / 3$ de l'intervalle, quel que soit le liquide et quelle que soit la polarité (Fig. 5). Ce minimum a été observé également par Chadband et Sufian dans d'autres liquides [7]. Ce profil de vitesses correspond à celui du champ électrique sur une sphère conductrice tangente à la pointe [8] pour les grandes vitesses et concentrique à la pointe [3] pour les autres cas (Fig. 5).

D'après cette étude, il apparaît que les processus électroniques jouent un rôle déterminant dans la propagation des streamers.

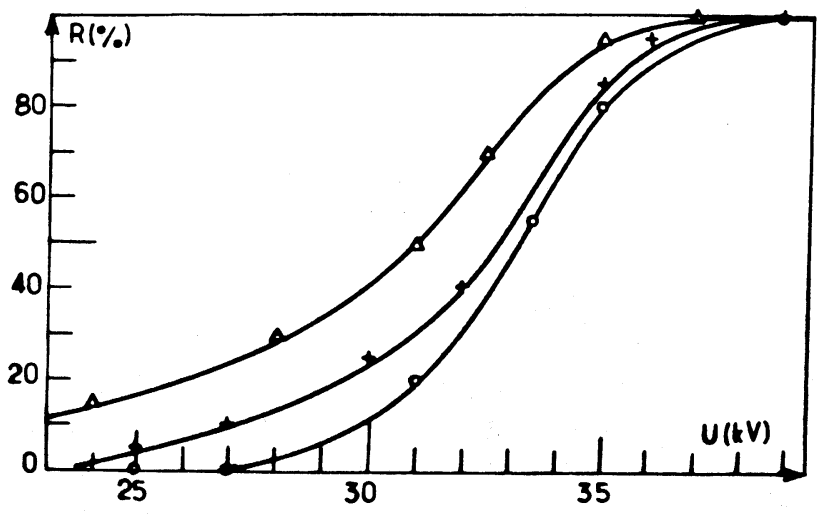

Fig. 6. - Probabilité d'apparition $(R)$ des streamers filamentaires (supersoniques) dans le cyclohexane en fonction de la tension appliquée pour une pointe négative dans diverses conditions de pression et de température. $L=$ $1,5 \mathrm{~mm} ; r_{\mathrm{p}}=3 \mu \mathrm{m}$. (O) $P=1$ bar ; $T=70^{\circ} \mathrm{C}$. (+) $P=1$ bar $; T=20^{\circ} \mathrm{C}$. $(\triangle) P=1,5$ bar $; T=20^{\circ} \mathrm{C}$.

[Appearance probabilité $(R)$ of filamentary streamers (supersonics) in cyclohexane as a function of the applied voltage for a negative point in different conditions of pressure and temperature. $L=1.5 \mathrm{~mm} ; r_{\mathrm{p}}=3 \mu \mathrm{m}$. (O) $P=1$ bar $; T=70^{\circ} \mathrm{C}$. (+) $P=1$ bar ; $T=20^{\circ} \mathrm{C}$. ( $\left.\triangle\right)$ $P=1.5$ bar $; T=20^{\circ} \mathrm{C}$.]

\section{Génération.}

Contrairement à la phase de propagation, il n'existe sur le plan expérimental, que très peu d'informations concernant la phase de génération des streamers. La 
plupart des études ont porté sur la mesure du temps de retard au claquage et sur celle des tensions disruptives ; les résultats ainsi obtenus ont plutôt un caractère industriel. L'étude de la phase de génération permettrait de progresser dans la compréhension des processus fondamentaux conduisant au claquage puisque c'est d'elle que va dépendre le déroulement des événements ultérieurs et c'est à propos des phénomènes qui s'y produisent que de nombreuses hypothèses ont été émises, d'où l'intérêt de connaître le temps et la tension de génération des streamers.

Le temps de générations $t_{\mathrm{g}}$ peut être évalué soit par les courbes de Von Laue $[9,10]$, soit en faisant varier le rayon de courbure $r_{\mathrm{p}}$ de la pointe et en mesurant le temps de retard au claquage pour une distance donnée [11]. En utilisant le même dispositif que précédemment, nous avons pu mettre au point une nouvelle méthode de détermination du temps et de la tension de génération des streamers.

Cette méthode consiste à déterminer la tension $\left(U_{\mathrm{g}}\right)$ et le temps $\left(t_{\mathrm{g}}\right)$ correspondant à l'apparition d'une petite perturbation $(10 \mu \mathrm{m}$ de diamètre environ) ou d'une émission lumineuse associée à cette perturbation. Selon la tension appliquée, le streamer peut apparaître soit sur le front de montée de la tension (si la pente de la rampe de tension est élevée) soit quand la tension est constante (si l'amplitude du créneau de tension est faible). Nous ne considérons ici, que le cas où le streamer est généré sur le plateau.

Dans ces conditions, le temps de génération $t_{\mathrm{g}}$ est compris entre $t_{\mathrm{m}}$ et $55 \mu \mathrm{s}\left(t_{\mathrm{m}}\right.$, temps de montée de la tension). La figure 7 , donne un exemple de variation de $t_{\mathrm{g}}=f(U)$ dans le cyclohexane. Nous remarquons que $t_{\mathrm{g}}$ est d'autant plus faible que l'amplitude du créneau de tension est élevée; $t_{\mathrm{g}+}$ et $t_{\mathrm{g}-}$ sont sensiblement les mêmes. Pour $\stackrel{U}{U}>U_{\mathrm{g}}$, la variation de $t_{\mathrm{g}}$ est lente $: t_{\mathrm{g}}$ est sensiblement proportionnel à $U^{-1}$ et $t_{\mathrm{g}} \rightarrow t_{\mathrm{m}}$. Pour des tensions plus élevées $(U>40 \mathrm{kV})$, la génération se produit durant la montée de la tension.

Nous avons observé que les temps de génération dans le PXE, le MDBT et les polybutènes (de viscosité variant de 90 à $4 \times 10^{5} \mathrm{cSt}$ à $20^{\circ} \mathrm{C}$ ), étaient du même ordre de grandeur que dans le cyclohexane.

D'autre part, si on identifie le temps de génération $t_{\mathrm{g}}$ au temps de retard $t_{\mathrm{r}}$ à la mise en mouvement du liquide induit par injection unipolaire de charges et que $t_{\mathrm{r}}$ est sensiblement le même qu'en géométrie plan-plan [12], celui-ci devrait être proportionnel à la viscosité $\eta$ du liquide $\left(t_{\mathrm{g}} \simeq t_{\mathrm{r}}=\right.$ Cte $\left.\eta / \varepsilon E^{2}\right)$; or, ce n'est pas le cas. Nous avons en effet procédé à l'étude des temps de retard à l'apparition des streamers pour les deux polarités dans les polybutènes cités ci-dessus, et nous n'avons pas observé d'augmentation ave $\eta$, ce qui semble exclure un mécanisme de cavitation induit par des phénomènes

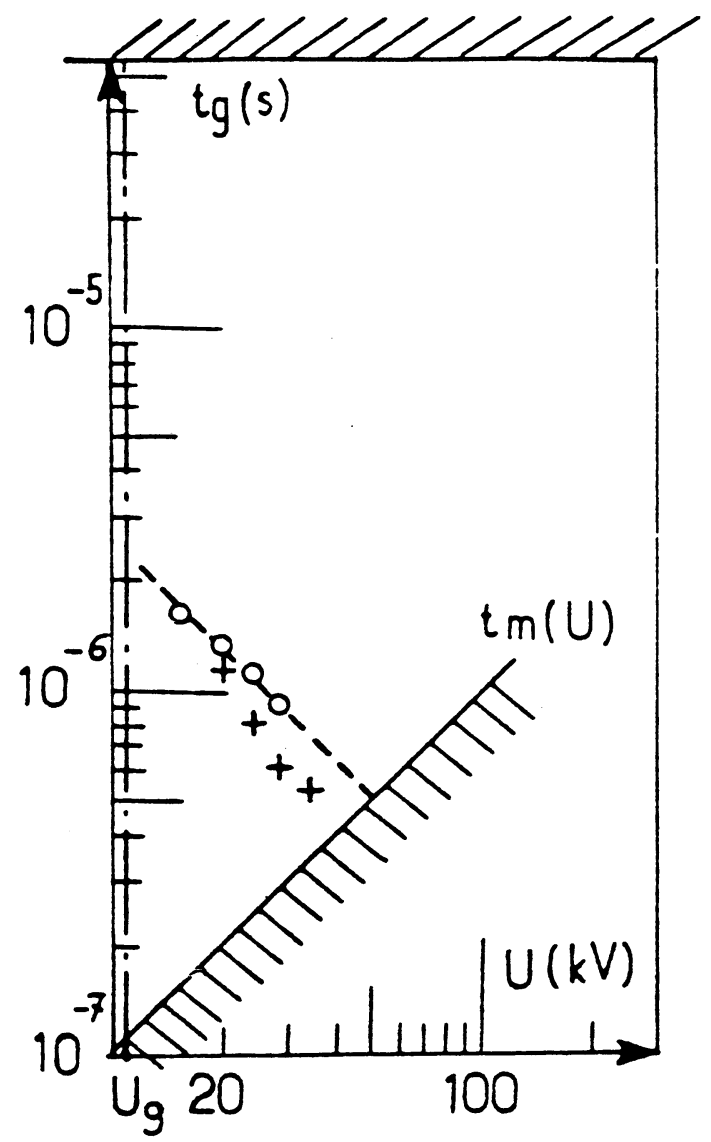

Fig. 7. - Variation du temps de génération des streamers dans le cyclohexane en fonction de l'amplitude du créneau de tension. $L=2 \mathrm{~mm} ; \eta_{0}=3 \mu \mathrm{m}$. (+) pointe positive. (O) pointe négative.

[Initiation time of streamers in cyclohexane as a function of the step voltage amplitude. $L=2 \mathrm{~mm} ; \eta_{0}=3 \mu \mathrm{m}$. $(+)$ positive point. $(O)$ negative point.]

électrohydrodynamiques (EHD) résultant d'une injection.

Quand à la tension de génération $U_{\mathrm{g}}$, elle croît avec le rayon de courbure de la pointe $r_{p}$ (Fig. 8a) alors qu'elle est peu sensible à la distance interélectrodes dans la gamme considérée $(0,5 \mathrm{~mm} \leqslant L \leqslant$ $6 \mathrm{~mm}$ ).

Le champ calculé en assimilant la pointe à un hyperboloïde $\left(E_{\mathrm{g}}=2 U_{\mathrm{g}} / r_{\mathrm{p}} \ln \left(4 L / r_{\mathrm{p}}\right)\right)$ pour $U$ et $L$ donnés, devrait varier approximativement en $\left(1 / r_{p}\right)$; or, le champ harmonique de génération déterminé à partir de $U_{\mathrm{g}}$ mesuré expérimentalement varie en $r_{\mathrm{p}}^{-n}(1 / 2<n \leqslant 2 / 3)$ (Fig. $\left.8 \mathrm{~b}\right)$.

On peut donc raisonnablement penser que pour les pointes à faible rayon de courbure $\left(r_{0}<10 \mu \mathrm{m}\right)$ le champ est affaibli par l'injection d'homocharges, alors que pour les pointes à rayon de courbure plus élevé, il serait localement renforcé par des irrégularités de surface. Rzad et al. [11] ont suggéré que ce serait le volume de liquide soumis au champ intense qui jouerait un rôle déterminant ce qui conduirait à une variation en $r_{\mathrm{p}}^{-1 / 3}$. 

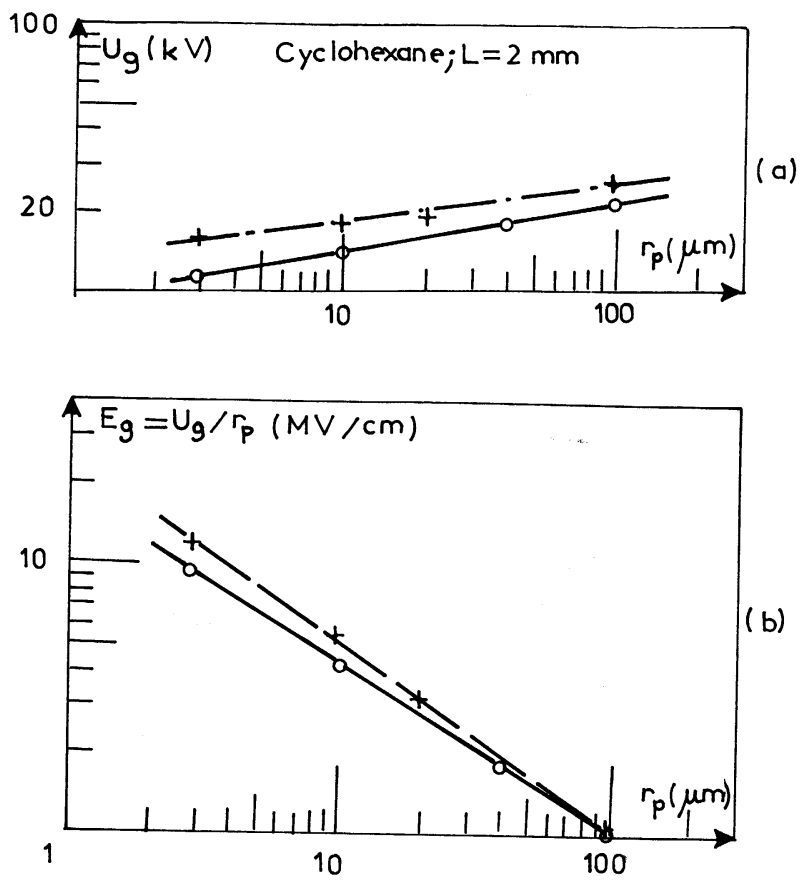

Fig. 8. - a) Tension de génération des streamers dans le cyclohexane en créneau de tension en fonction du rayon de courbure de la pointe pour les deux polarités : $(+)$ pointe positive. (O) pointe négative. b) Champ harmonique de génération déduit des valeurs précédentes de $U_{\mathrm{g}}$ avec l'approximation hyperboloïdale.

(a) Initiation voltage of streamers in cyclohexane under step voltage versus the tip radius of the needle in both polarities: $(+)$ positive point and $(0)$ negative point. b) Initiation harmonic field deduced from the above values of $U_{\mathrm{g}}$ in hyperboloid approximation case.]

\section{Influence de la pression.}

La figure 9, montre l'influence de la pression hydrostatique sur la forme du streamer dans le cyclohexane en pointe négative $\left(U=28 \mathrm{kV}, t_{\mathrm{m}}=0,3 \mu \mathrm{s}, L=\right.$ $1 \mathrm{~mm}, r_{\mathrm{p}}=5 \mu \mathrm{m}$ ) dans le régime de propagation lent. Les photographies sont prises $7 \mu \mathrm{s}$ après application de la tension. On remarque que sous l'effet d'une pression modeste, le streamer disparaît. Il en est de même du courant et de la lumière émise par le streamer. L'effet est le même pour les streamers rapides sauf qu'il faut plus de pression pour leur disparition que pour les streamers lents. Nous avons observé le même phénomène dans d'autres liquides (PXE, MDBT, huile minérale, huile silicone, cyclohexane $\left.+1 \% \mathrm{CCl}_{4}\right)$. La pression nécessaire à la disparition du streamer $P_{\text {disp. }}$ est d'autant plus importante que la tension est élevée et donc la vitesse est grande. La figure 10 représente la variation de $P_{\text {disp. }}$ en fonction de la tension appliquée $U$ dans le cyclohexane, le PXE et le MDBT, pour les deux polarités. La variation est sensiblement linéaire, ce qui évoque la loi de Paschen au-delà du minimum.

La pression joue donc un rôle similaire sur les

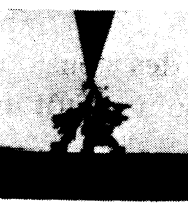

$1.0 \mathrm{bar}$

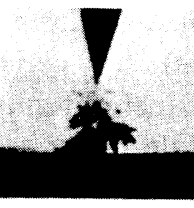

1.1

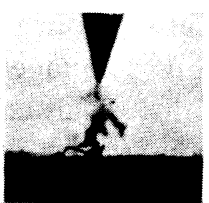

1.3

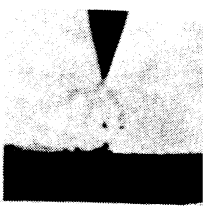

1.5
Fig. 9. - Streamer négatif dans le cyclohexane sous l'effet de la pression. $U=28 \mathrm{kV} ; L=1 \mathrm{~mm} ; r_{\mathrm{p}}=$ $5 \mu \mathrm{m}$. Toutes les photographies sont prises $7 \mu \mathrm{s}$ après application de la tension.

[Influence of the pressure on the shape of the negative streamer in cyclohexane $U=28 \mathrm{kV} ; L=1 \mathrm{~mm} ; r_{\mathrm{p}}=$ $5 \mu \mathrm{m}$. All photographs were taken $7 \mu \mathrm{s}$ after beginning of the voltage rise.]

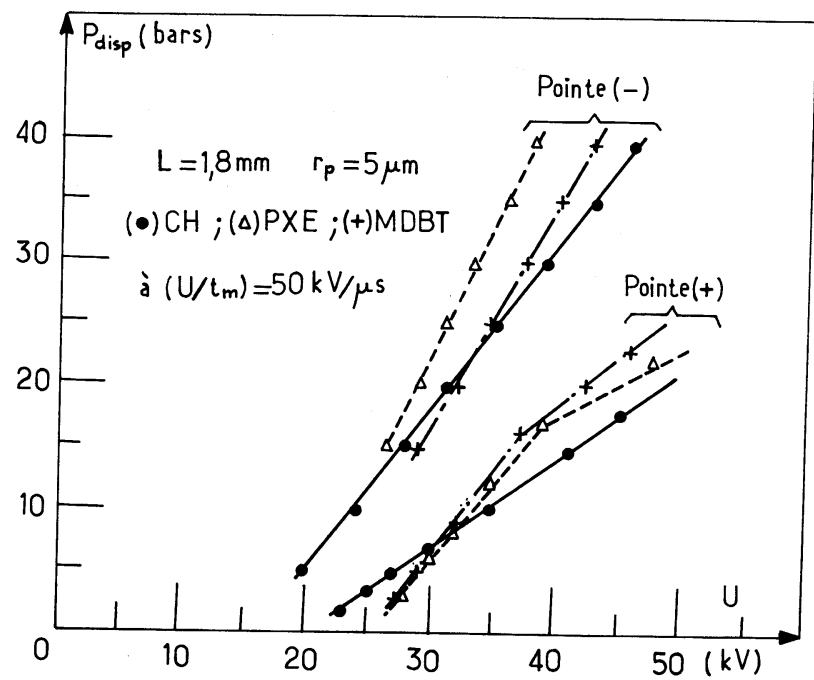

Fig. 10. - Caractéristiques $P_{\text {disp. }}=f(U)$ dans le cyclohexane, le PXE et le MDBT dans les deux polarités.

[Characteristics $P_{\text {disp. }}=f(U)$ in cyclohexane, PXE and MDBT for both polarities.]

deux polarités ce qui indique l'existence d'une phase gazeuse dans les deux cas, contrairement aux résultats de Yoshino [9] selon lesquels en polarité négative, la tension de claquage de l'azote liquide croît continûment quand $\boldsymbol{P}$ augmente alors qu'en polarité positive, la croissance est bien moins rapide et stoppe au-delà de $10 \mathrm{~atm}$. Cette phase gazeuse pourrait être produite soit par cavitation, soit par ébullition ou encore par dissociation des molécules du liquide. Si l'apparition de la phase gazeuse est attribué à la cavitation due aux écoulements électrohydrodynamique (EHD) induits par l'injection unipolaire de charges dans le liquide, la variation de la pression serait, en vertu du théorème de Bernouilli :

$$
\Delta p=(1 / 2) \rho v_{\mathrm{EHD}}^{2}
$$

où $\rho$ et $v_{\mathrm{EHD}}$, sont respectivement la masse volumique et la vitesse EHD du liquide. 
Comme

$$
\begin{aligned}
v_{\mathrm{EHD}} & \sim \sqrt{\varepsilon / \rho} E, \\
\Delta p & \simeq(1 / 2) \varepsilon E^{2}
\end{aligned}
$$

$\Delta p$, serait proportionnelle à $E^{2}$ et donc à $U^{2}$, ce qui n'est pas le cas.

\section{Conclusion.}

Il existe une corrélation très étroite entre la forme du streamer, sa vitesse de propagation, la forme du courant et la lumière émise qui l'accompagne indépendamment du liquide et de la polarité de la pointe. La distribution de vitesse admet toujours un minimum plus ou moins marqué compris entre le milieu et les $2 / 3$ de l'intervalle quelle que soit l'amplitude des vitesses; sa variation suit celle du champ sur une sphère conductrice se développant de la pointe vers le plan.

Quelle que soit la polarité, la pression agit sur l'apparition du streamer, ce qui indique que des processus gazeux dominent et non les processus électroniques comme celà a été avancé en polarité positive. Cependant, au vu des résultats expérimentaux concernant l'effet de la viscosité sur le temps de génération des streamers ainsi que de la variation de la tension de génération en fonction de la pression hydrostatique, le mécanisme de cavitation semble être écarté.

\section{Bibliographie}

[1] Devins, J. C., Rzad, S. J. and Schwabe, R. J., J. Appl. Phys. 52 (1981) 4531.

[2] Sakamoto, S. and Yamada H., I.E.E.E. Trans. Electr. Insul. EI-15 (1980) 171.

[3] Beroual, A. and Tobazeon, R., I.E.E.E. Trans. Electr. Insul. EI-21 (1986) 613.

[4] Yamashita, H. and Amano, H., I.E.E.E. Trans. Electr. Insul. EI-20 (1985) 247.

[5] YamadA, H. and SATO, T., I.E.E.E. Trans. Electr. Insul. EI-20 (1985) 261.

[6] MCKenny, P. J. and MCGrath, P. B., I.E.E.E. Trans. Electr. Insul. EI-19 (1984) 93.
[7] Chadband, W. G. and Sufian, T. M., I.E.E.E. Trans. Electr. Insul. EI-20 (1985) 239.

[8] Chadband, W. G. and Wright, G. T., Br. J. Appl. Phys. 16 (1965) 305.

[9] Yoshino, K., I.E.E.E. Trans. Electr. Insul. EI-15 (1980) 186.

[10] ARII, K., HAYASHI, K., KITANI, I. and INUISHI, Y., Proc. 5th Int. Conf. Cond. Breakd. Diel. Liq., Delft (1975) p. 163.

[11] Rzad, S. J., Devins, J. C. and Schwabe, R. J., I.E.E.E. Trans. Electr. Insul. EI-14 (1979) 289.

[12] Tobazeon, R., J. Electrostatics 15 (1984) 359. 